\title{
Frequency, comprehension and attitudes of physicians towards abbreviations in the medical record
}

\author{
Uri Hamiel, ${ }^{1,2}$ Idan Hecht, ${ }^{2,3}$ Achia Nemet, ${ }^{2,3}$ Liron Pe'er, $^{2,3}$ Vitaly Man, ${ }^{4}$ Assaf Hilely, ${ }^{5}$ \\ Asaf Achiron ${ }^{2,3}$
}

${ }^{1}$ Department of Pediatrics, Assaf Harofeh Medical Center, Zerifin, Israel

${ }^{2}$ Sackler Faculty of Medicine, Tel Aviv University, Tel Aviv, Israel ${ }^{3}$ Department of Ophthalmology, Edith Wolfson Medical Center, Holon, Israel

${ }^{4}$ Department of Ophthalmology, Soroka University Medical

Center, Ben-Gurion University, Beer Sheva, Israel

${ }^{5}$ Department of Ophthalmology, Kaplan Medical Center, Rehovot and the Hebrew University of Jerusalem-Hadassah Medical School, Jerusalem, Israel

Correspondence to Dr Uri Hamiel, Department of Pediatrics, Assaf Harofeh Medical Center, Zerifin 70300 Israel; urihamiel@gmail.com

Received 13 December 2017 Revised 12 February 2018 Accepted 24 February 2018 Published Online First 14 March 2018

\section{ABSTRACT}

Aims Abbreviations are common in the medical record. Their inappropriate use may ultimately lead to patient harm, yet little is known regarding the extent of their use and their comprehension. Our aim was to assess the extent of their use, their comprehension and physicians' attitudes towards them, using ophthalmology consults in a tertiary hospital as a model.

Methods We first mapped the frequency with which English abbreviations were used in the departments' computerised databases. We then used the most frequently used abbreviations as part of a crosssectional survey designed to assess the attitudes of non-ophthalmologist physicians towards the abbreviations and their comprehension of them. Finally, we tested whether an online lecture would improve comprehension.

Results 4375 records were screened, and 235 physicians responded to the survey. Only $42.5 \%$ knew at least $10 \%$ of the abbreviations, and no one knew them all. Ninety-two per cent of respondents admitted to searching online for the meanings of abbreviations, and $59.1 \%$ believe abbreviations should be prohibited in medical records. A short online lecture improved the number of respondents answering correctly at least $50 \%$ of the time from $1.2 \%$ to $42 \%(P<0.001)$.

Conclusions Abbreviations are common in medical records and are frequently misinterpreted. Online teaching is a valuable tool for physician education. The majority of respondents believed that misinterpreting abbreviations could negatively impact patient care, and that the use of abbreviations should be prohibited in medical records. Due to low rates of comprehension and negative attitudes towards abbreviations in medical communications, we believe their use should be discouraged.

\section{INTRODUCTION}

Abbreviations, which are defined as a shortened form of a word or name, are common in the medical records. ${ }^{1}$ A study which looked at the electronic discharge summaries from a large, tertiary teaching hospital in Australia revealed that abbreviations were common, occurring at a frequency of one in five words. ${ }^{2}$ Other studies have also found widespread use of abbreviations in other types of medical records. ${ }^{3-5}$

A UK study from 2008 checked a selection of abbreviations from surgical inpatient admissions and found that the majority of healthcare professionals have very poor knowledge of commonly used abbreviations. Comprehension of the abbreviations varied from 20\% (dieticians) to $57 \%$ (young doctors). ${ }^{6}$ Some abbreviations are referred to as 'dangerous' or 'error-prone' because they can lead to misinterpretation of orders and other communications, resulting in patient harm. ${ }^{7}$ A study carried out in a few Australian hospitals determined that $76.9 \%$ of patients had one or more error-prone abbreviations in their medical prescriptions, with $29.6 \%$ of these considered by the authors to be of high risk for causing significant harm. ${ }^{8}$ Prescription errors caused by the misinterpretation of abbreviations were found in about $3 \%$ of both handwritten and preprinted prescriptions in an ophthalmology clinic in Thailand. ${ }^{9}$ The inappropriate use of jargon can lead to confusion and miscommunication between the specialist and the physician seeking consult, as well as with the patient, potentially leading to misdiagnosis or improper treatment. ${ }^{10}$ Educational interventions were previously found to markedly reduce the use of unsafe abbreviations in medication orders. ${ }^{11}$

In Israel, the language most commonly used in written medical consultations is Hebrew, yet English-language abbreviations are very common and are prevalent in certain fields such as ophthalmology. ${ }^{12}$ In this study, we intended to evaluate which abbreviations are most frequently used by ophthalmologists in a tertiary hospital in Israel and examine the attitudes of general practitioners (GPs) towards them. We then checked which abbreviations are poorly understood, and, finally, tested whether a short online lecture could improve understanding of these abbreviations.

\section{METHODS}

\section{Abbreviation use analysis}

We conducted a computerised query on the Edith Wolfson Medical Center's ophthalmology department's database. As anonymisation and transfer of the digital documents for analysis required manually intensive technical work, we randomly selected those belonging to five residents and one senior physician (mean years of experience: $3.5 \pm 2.2$ ) as a representative sample of the department's physicians. The documents included all electronic emergency department visit summaries, specialty consultations and discharge letters in the years 2003-2017.

We then proceeded to create a common abbreviations list using a Delphi method. ${ }^{13}$ Our Delphi method included three rounds and seven panellists from several subspecialties, and was performed in accordance with the methods laid out by Hsu et al. ${ }^{14}$ In the first round, a general list of all abbreviations 


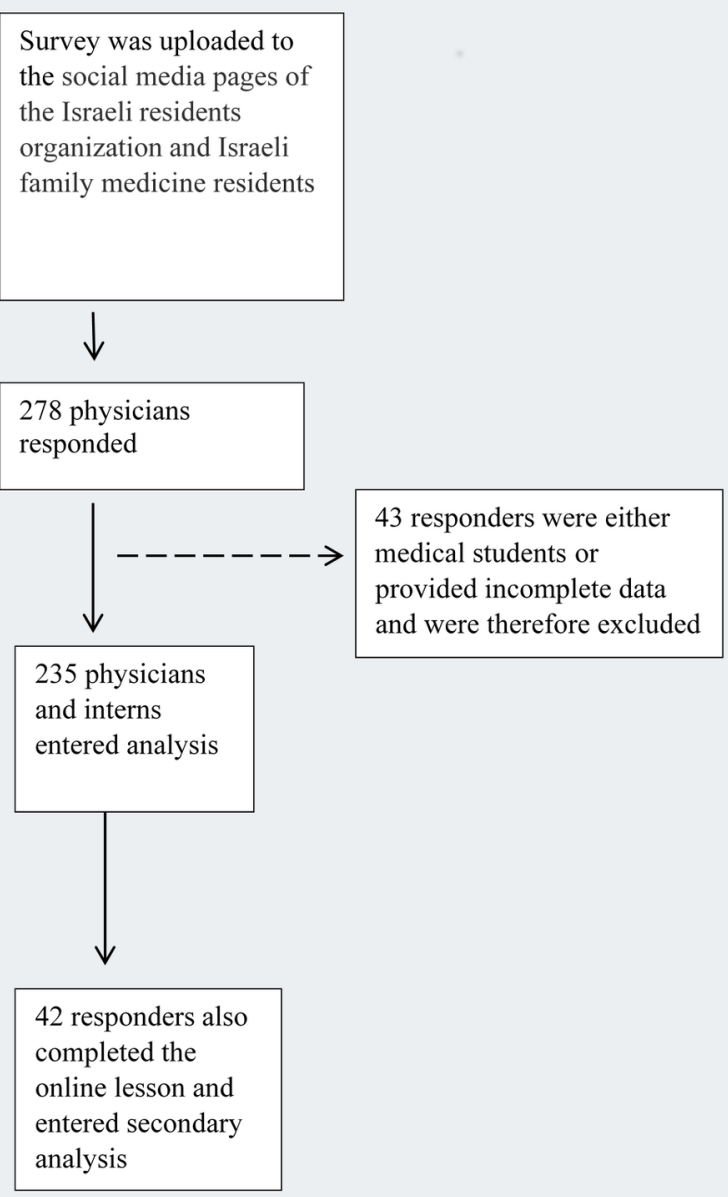

Figure 1 Flow chart of the inclusion process.

used in ophthalmology was created. In the second round, panellists ranked the items on the list according to how commonly they are used in ophthalmic clinics and how often they must be explained to patients or primary care providers. In the final round, the list of items and their rankings was returned to the panellists to give them an opportunity to revise their judgements. We then searched for documents containing the ophthalmic abbreviations. Last, we analysed each document, counted the number of times each abbreviation occurred and mapped them according to frequency.

\section{Survey questionnaire}

We used the 18 most frequent abbreviations as part of an anonymous cross-sectional survey designed to assess respondents' attitudes towards and comprehension of commonly used ophthalmological abbreviations. The survey was limited to 18 of the most commonly used abbreviations in order to ensure a high response rate. It was conducted over 2 weeks in July 2017, among two populations: (1) non-ophthalmologist physicians employed at our institute and (2) interns, residents and senior physicians using the social media pages of the Israeli residents' organisation and Israeli family medicine residents (figure 1). We asked for an email address to ensure that each person only submitted one entry. The abbreviations used in the survey are shown in table 1. Inclusion criteria were as follows: (1) physicians currently practising medicine and (2) non-ophthalmologists. Respondents' interpretations of abbreviations on the survey were marked as
Table 1 Common ophthalmological abbreviations, their complete forms and correct interpretation rates in the study population

\begin{tabular}{|c|c|c|c|}
\hline Abbreviation & Correct term & $\begin{array}{l}\text { Most common } \\
\text { wrong interpretation }\end{array}$ & $\begin{array}{l}\text { Correct } \\
\text { answers as a } \\
\text { per cent } \\
\text { of all answers }\end{array}$ \\
\hline OCT & $\begin{array}{l}\text { Optical coherence } \\
\text { tomography }\end{array}$ & $\begin{array}{l}\text { Computerised } \\
\text { tomography }\end{array}$ & 20.9 \\
\hline VA & Visual acuity & Ventriculo-atrial & 10.7 \\
\hline HM & Hand motion & & 2.1 \\
\hline $\mathrm{CF}$ & Counting fingers & Cystic fibrosis & 1.7 \\
\hline VF & Visual field & Ventricular fibrillation & 8.5 \\
\hline AMD & $\begin{array}{l}\text { Age-related macular } \\
\text { degeneration }\end{array}$ & & 48.5 \\
\hline PHACO & Phacoemulsification & & 16.6 \\
\hline KP & Keratic precipitate & & 1.7 \\
\hline SVP & $\begin{array}{l}\text { Spontaneous venous } \\
\text { pulsation }\end{array}$ & & 1.7 \\
\hline FBE & Fundus both eyes & Full blood examination & 3.0 \\
\hline FRE & Fundus right eye & & 2.6 \\
\hline FLE & Fundus left eye & Frontal lobe epilepsy & 2.6 \\
\hline ONHD & Optic nerve head drusen & & 0 \\
\hline RAPD & $\begin{array}{l}\text { Relative afferent } \\
\text { pupillary defect }\end{array}$ & & 30.3 \\
\hline PDR & $\begin{array}{l}\text { Proliferative diabetic } \\
\text { retinopathy }\end{array}$ & & 6.8 \\
\hline CME & Cystoid macular edema & & 0.9 \\
\hline PXF & Pseudoexfoliation & & 1.3 \\
\hline IOL & Intra-ocular lens & & 10.3 \\
\hline
\end{tabular}

either correct or incorrect. We then used a subset of the survey population, who agreed to participate when they filled out the primary survey, to test whether a short online lecture could improve understanding of these abbreviations. They were asked to take an online lecture and repeat the original survey a week later. Participation was voluntary, and no reference materials were permitted.

\section{Statistical analysis}

Statistical analysis was performed using IBM SPSS Statistics V.22.0 for Windows. P values $<0.05$ on a two-sided test were considered statistically significant. We conducted $\chi^{2}$ tests for categorical variables. Clinical parameter distributions were tested for normality using the Shapiro-Wilk test. Independent and paired t-tests were conducted for continuous variables with normal distributions, and the Wilcoxon signed-rank and Mann-Whitney $U$ tests were done for variables with non-normal distributions. Unless otherwise specified, data are presented as mean \pm SD.

\section{RESULTS}

\section{Abbreviation use analysis}

We analysed 4375 records and identified 54 abbreviated medical terms. Of all documents, 2993 (68.4\%) contained at least one abbreviation. The three most frequent abbreviations were 'IOL' (1279 occurrences, $29.2 \%$ of documents contained this abbreviation), 'OCT' (1006 occurrences, 22.9\%), 'SPK' (838 occurrences, $19.15 \%)$.

\section{Survey questionnaire}

The survey was filled in by 278 respondents. Forty-three were either medical students or provided incomplete data, and were 


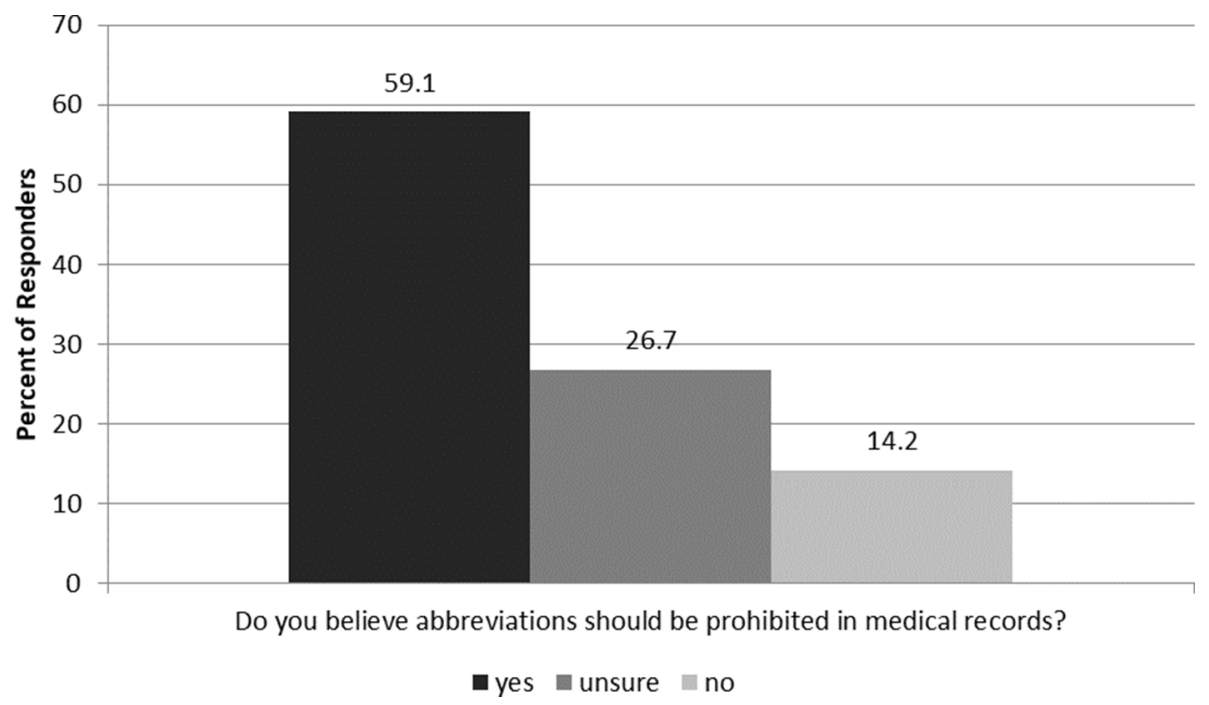

Figure 2 Physicians' opinion on banning abbreviations.

therefore excluded. The survey questionnaire was completed by 235 physicians and interns. The median age of respondents was 33 (IQR 5), and 61.1\% were female. Of the 235 respondents whose answers we analysed, 59.1\% were residents, 20\% were senior doctors, $2.1 \%$ were GPs without a specialty and $18.7 \%$ were interns. The physicians' specialties were (in descending order): general practice $(20.0 \%)$, internal medicine $(18.5 \%)$, paediatrics $(18.0 \%)$, obstetrics-gynaecology (5.5\%), psychiatry $(5.0 \%)$ and other specialties (33\%). The median number of years in clinical practice was 3 (IQR 5.5). Eighty-three per cent of physicians said that they refer patients to ophthalmologists at least once a month.

\section{Abbreviation comprehension and attitudes towards them}

No respondent correctly interpreted all 18 of the abbreviations on the survey. Only $1.2 \%$ knew at least $50 \%(9 / 18)$ of the abbreviations, $9.7 \%$ of respondents knew at least $25 \%$ (4 of 18) and $42.5 \%$ correctly identified at least $10 \%(2 / 18)$ of the terms. $37.4 \%$ of respondents failed to correctly identify any of the abbreviations.

The abbreviations 'AMD', 'RAPD' and 'OCT' were the most often correctly interpreted, and 'ONHD' and 'CME' were the least. Further details regarding abbreviation comprehension and common incorrect interpretations are found in table 1.

A majority (79\%) of respondents believed it was either important or very important for them to know the meanings of the abbreviations, 19\% thought there was some importance and only $2.1 \%$ saw no importance in knowing the abbreviated terms. Ninety per cent thought that knowing these terms would, to some degree, improve the quality of the care they provide. Ninety-three per cent of respondents claimed to get 'irritated' or 'very irritated' when they encounter an abbreviation they are not familiar with. Almost all (92.3\%) admitted to having used a search engine like Google to find the meaning of an abbreviation. Finally, 59.1\% believe abbreviations should be prohibited in medical consultations and records, $26.7 \%$ are undecided and $14.2 \%$ do not think abbreviations should be banned (figure 2).

\section{Online lesson on ophthalmic abbreviations}

Of the 235 who completed the first survey, 42 (18\%) completed the online class and the subsequent exam. Following the online lesson, the percentage of respondents who scored at least $25 \%$ improved from $9.7 \%$ to $67 \%(\mathrm{P}<0.05)$. The percentage

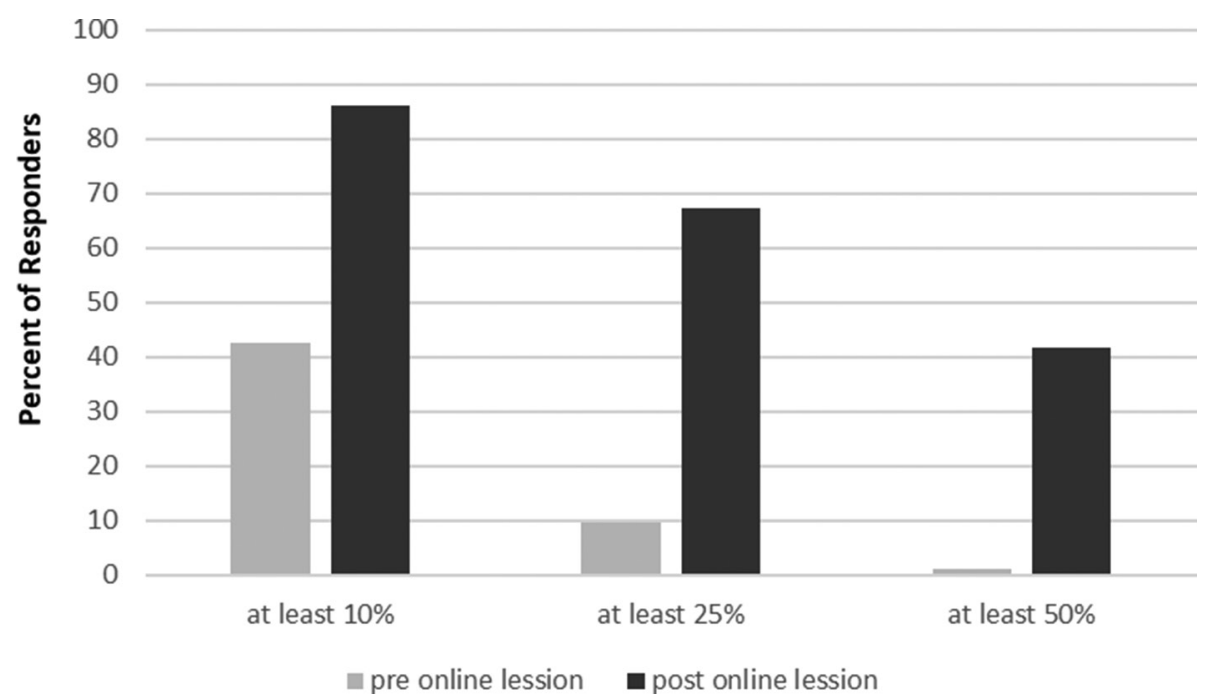

Figure 3 Proportion of correct answers. 
of respondents who got at least $50 \%$ improved from $1.2 \%$ to $42 \%(\mathrm{P}<0.05)$, as illustrated in figure 3. Of the participants who completed the online class, $65.9 \%$ responded that it was a convenient way to improve knowledge and $41.5 \%$ felt their knowledge actually improved.

To note, those who chose to take part in the online course had slightly better results at baseline (mean correct answers: $2.60 \pm 2.0$ vs $1.68 \pm 2,13, \mathrm{P}=0.04$; percentage who correctly knew at least $10 \%$ of abbreviations: $27.2 \%$ vs $11.9, \mathrm{P}=0.02$ ) However, no differences were observed in the percentages of subjects who knew at least $25 \%$ or $50 \%$ as these rates were very low $(\mathrm{P}=0.9$ for both comparisons).

\section{DISCUSSION}

Many factors contribute to the widespread use of medical abbreviations: time constraints in the workplace, the need to provide digital documentation and the standard practice of abbreviating text for other formats, such as social media and instant messaging. ${ }^{2}$ The United States Medical Licensing Examination, mandatory for all physicians wanting to practice in the USA, even allows test takers to use commonly used abbreviations on the clinical skills section. ${ }^{15}$ It is easy to understand why abbreviations are common in medicine.

As more doctors go into specialised medicine, there is a corresponding increase in the number of specialty consultations in ambulatory care and hospital settings. ${ }^{16}$ Doctors and patients should therefore be able to read and understand the consultations without needing clarification. ${ }^{17}$ Still, many specialists use abbreviations which are either not universally accepted or are indecipherable to physicians outside their specialties. The use of ambiguous abbreviations is also common. ${ }^{18}$ Developers have even written algorithms to correctly interpret the abbreviations in clinical notes. ${ }^{19} 20$

The use of abbreviations can lead to miscommunication, confusion and patient harm. ${ }^{7-10}$ An earlier publication demonstrated that most physicians who were not dermatologists knew fewer than $25 \%$ of commonly used dermatology abbreviations. ${ }^{21}$ It is important to mention that staff were more likely to correctly interpret abbreviations commonly used within their own fields. ${ }^{6}$ The only reference in the literature to ophthalmological abbreviations is a small study with 48 British GPs. They answered a questionnaire about 12 ophthalmological abbreviations, and $67 \%$ of their answers were wrong. ${ }^{22}$

In our study, we used a computerised query to identify the abbreviations most commonly used by ophthalmologists. We then used crowdsourcing to find survey participants.

Previous studies have shown that crowdsourcing is an efficient and reliable method for collecting responses, offering an appropriate alternative to traditional surveying. ${ }^{23-25}$ This method enables a researcher to involve many more participants than by traditional methods (printed questionnaires), but without the option of reporting on response rate.

Of our survey's respondents, only $1.2 \%$ knew how to correctly interpret half of the abbreviations, and fewer than $10 \%$ knew the correct meanings of a quarter of the abbreviations. Nearly $98 \%$ of respondents believed that knowing the correct meanings of the abbreviations was important, and almost 90\% thought that understanding the abbreviations would improve the quality of the care they provided. At the end of our study, we show how a short online lecture can teach doctors to correctly interpret abbreviations.

This study has a number of limitations. First, our calculations of each abbreviation's rate of occurrence is based solely on retrospective chart reviews for the ophthalmology department of a single hospital. Their usage may vary in other settings. Second, the abbreviations were given out of context in the survey, which is not how they would likely appear in medical records. Third, participation in the survey and online lesson was voluntary, and so a selection bias may have existed. In addition, when we assessed the benefits of taking the lesson, a difference (0.91 in mean correct answers) was found in favour of those who opted to participate in that second part of the survey. Despite the differences being small, it may still affect our results due to a participation bias. ${ }^{26}$ Finally, no mechanism existed to prevent the physicians surveyed from using reference materials, though it was written in the instructions not to.

In conclusion, abbreviations were frequent in the medical records we used, but only a small proportion of physicians could correctly interpret the most common of them. Over $90 \%$ of respondents admitted to using web searches to find the meanings of abbreviations on the job, and nearly $60 \%$ believed abbreviations should be prohibited in medical consultations and records. None of the respondents could correctly interpret all 18 of the most common abbreviations. The majority of them believed misunderstanding abbreviations could harm patient care. Comprehension of abbreviations improved after watching a short lecture online. Due to the low comprehension rate and negative attitudes towards abbreviations in medical communications, their use should be highly discouraged. The misinterpretation of abbreviations might hinder doctors' efforts to provide the highest quality healthcare to their patients. If time restraints and work habits do not allow for abbreviations to be eliminated from use, a printed reference list of common ones should be incorporated into the medical record.

\section{Main messages}

- Abbreviations are common in medical records and are frequently misinterpreted.

- Physicians believe misunderstanding of abbreviations could harm patient care and that abbreviations should be prohibited in medical records.

- Online teaching is a valuable tool in physicians' continuing education.

\section{Current research questions}

- Should abbreviations be banned altogether from medical records?

- Which interventions could minimise abbreviation use in medical records?

- Which tools could be developed to limit interdisciplinary misunderstanding between physicians regarding the written medical record?

Acknowledgements The authors thank Ms Danielle Meyerson for her excellent editorial assistance.

Contributors $\mathrm{UH}, \mathrm{IH}$ and AA led the study, were responsible for the study design, supervised the collection of data and analysis, and are the guarantors of the article. AN, LP, VM and AH were involved in data collection and helped with the literature review. IH and $A A$ analysed the data. $U H, A N, L P, V M$ and $A H$ reviewed the data analysis. $U H, I H$ and $A A$ wrote the majority of the paper and were assisted by $A N, L P$, VM and AH. All authors approved the final version of the manuscript.

Funding The authors have not declared a specific grant for this research from any funding agency in the public, commercial or not-for-profit sectors.

\section{Competing interests None declared.}

Patient consent Detail has been removed from this case description/these case descriptions to ensure anonymity. The editors and reviewers have seen the detailed information available and are satisfied that the information backs up the case the authors are making.

Ethics approval The study was performed following all the guidelines for experimental investigations required by the Institutional Review and Ethics Committee of the Edith Wolfson Medical Center. 
Provenance and peer review Not commissioned; externally peer reviewed.

Data sharing statement Raw data of this study are available by personal request to the authors.

(c) Article author(s) (or their employer(s) unless otherwise stated in the text of the article) 2018. All rights reserved. No commercial use is permitted unless otherwise expressly granted.

\section{REFERENCES}

1 Abbreviation. Definition of Abbreviation by Merriam-Webster. https://www.merriamwebster.com/dictionary/abbreviation (accessed 25 Jun 2017).

2 Politis J, Lau S, Yeoh J, et al. Overview of shorthand medical glossary (OMG) study. Intern Med J 2015;45:423-7.

3 Sheppard JE, Weidner LC, Zakai S, et al. Ambiguous abbreviations: an audit of abbreviations in paediatric note keeping. Arch Dis Child 2008;93:204-6.

4 Manzar S, Nair AK, Govind Pai M, et al. Use of abbreviations in daily progress notes. Arch Dis Child Fetal Neonatal Ed 2004;89:F374.

5 Collard B, Royal A. The use of abbreviations in surgical note keeping. Ann Med Surg 2015;4:100-2.

6 Sinha S, McDermott F, Srinivas G, et al. Use of abbreviations by healthcare professionals: what is the way forward? Postgrad Med J 2011;87:450-2.

7 Koczmara C, Jelincic V, Dueck C. Dangerous abbreviations: "U" can make a difference!. Dynamics 2005;16:11-15.

8 Dooley MJ, Wiseman M, Gu G. Prevalence of error-prone abbreviations used in medication prescribing for hospitalised patients: multi-hospital evaluation. Intern Med J 2012;42:e19-22.

9 Sanguansak T, Morley MG, Yospaiboon Y, et al. The impact of preprinted prescription forms on medication prescribing errors in an ophthalmology clinic in northeast Thailand: a non-randomised interventional study. BMJ Open 2012;2:e000539.

10 Brunetti L, Santell JP, Hicks RW. The impact of abbreviations on patient safety. Jt Comm J Qual Patient Saf 2007;33:576-83.

11 Abushaiqa ME, Zaran FK, Bach DS, et al. Educational interventions to reduce use of unsafe abbreviations. Am J Health Syst Pharm 2007;64:1170-3.
12 Shilo G, Shilo L. Writing style of young physicians in the computer and internet era. Int J Med Educ 2014;5:82-6.

13 de Villiers MR, de Villiers PJ, Kent AP. The Delphi technique in health sciences education research. Med Teach 2005;27:639-43.

14 Hsu C-C, Sandford BA. The Delphi Technique: Making Sense of Consensus. Pract Assessment Res Eval 2007;12:1-8.

15 Federation of State Medical Boards of the United States, Inc and the NB of ME. Common abbreviations for the patient note, Step 2 Clinical Skills (CS). $2017 \mathrm{http}: / /$ www.usmle.org/pdfs/step-2-cs/cs-info-manual.pdf (accessed 25 Jun 2017).

16 Jordan MR, Conley J, Ghali WA. Consultation patterns and clinical correlates of consultation in a tertiary care setting. BMC Res Notes 2008;1:96.

17 Lee EH, Patel JP, Fortin AH. Patient-centric medical notes: Identifying areas for improvement in the age of open medical records. Patient Educ Couns 2017; 100:1608-11.

18 Lövestam E, Velupillai S, Kvist M. Abbreviations in Swedish Clinical Text--use by three professions. Stud Health Technol Inform 2014;205:720-4.

$19 \mathrm{Xu} \mathrm{H}, \mathrm{Wu}$ Y, Elhadad N, et al. A new clustering method for detecting rare senses of abbreviations in clinical notes. J Biomed Inform 2012;45:1075-83.

20 Pakhomov S, Pedersen T, Chute CG. Abbreviation and acronym disambiguation in clinical discourse. AMIA Annu Symp Proc 2005:589-93.

21 Vale SM, Koenig K, Ailor SK, et al. Knowledge of dermatologic abbreviations: a survey of patients and physicians who are not dermatologists. J Am Acad Dermatol 2017;76:362-4.

22 Ali N, Khan AA, Akunjee M, et al. Using common ophthalmologic jargon in correspondence can lead to miscommunication. Br J Gen Pract 2006;56:968-9.

23 Ranard BL, Ha YP, Meisel ZF, et al. Crowdsourcing--harnessing the masses to advance health and medicine, a systematic review. J Gen Intern Med 2014;29:187-203.

24 Behrend TS, Sharek DJ, Meade AW, et al. The viability of crowdsourcing for survey research. Behav Res Methods 2011;43:800-13.

25 Brabham DC, Ribisl KM, Kirchner TR, et al. Crowdsourcing applications for public health. Am J Prev Med 2014;46:179-87.

26 Arruda-Olson AM, Weston SA, Fridley BL, et al. Participation bias and its impact on the assembly of a genetic specimen repository for a myocardial infarction cohort. Mayo Clin Proc 2007;82:1185-91 